\title{
Bioseguridad y medidas de protección de las enfermeras en el servicio de neonatología del Hospital Regional Docente Materno Infantil El Carmen de Huancayo
}

\section{Biosecurity and protection measures for nurses in the neonatology service of the Regional Maternal and Child Teaching Hospital El Carmen of Huancayo}

\author{
Amélida V. Rupay A. ("), Karla Cruz R.(1) y Patricia Zambrano E.(1) \\ (1)Facultad de Enfermería de la Universidad Nacional del Centro del Perú
}

Email: arupay@uncp.edu.pe

\begin{abstract}
RESUMEN
La investigación titulada Bioseguridad y medidas de protección de las enfermeras en el servicio de neonatología del Hospital Regional Docente Materno Infantil El Carmen de Huancayo parte del siguiente problema: ¿En qué medida el nivel de conocimiento sobre bioseguridad se relaciona con el uso de medidas de protección de las enfermeras en el Servicio de Neonatología del Hospital Docente Materno Infantil “El Carmen” de Huancayo?; el objetivo que persigue es: Determinar la relación entre el nivel de conocimiento sobre bioseguridad y el uso de medidas de protección de las enfermeras en el Servicio de Neonatología del Hospital Docente Materno Infantil "El Carmen" de Huancayo. El diseño de investigación es correlacional, nivel explicativo, tipo básico, el método utilizado fue el histórico y descriptivo. La muestra está conformada por 40 enfermeras(os).

Los resultados de la investigación social - empírica, nos han permitido elaborar conclusiones importantes; como que hay un alto conocimiento sobre medidas de bioseguridad por parte de las enfermeras del Servicio de Neonatología del Hospital Docente Materno Infantil "El Carmen" de Huancayo. Este nivel de conocimiento, sobre medidas de bioseguridad, se encuentra relacionada significativamente con el empleo de medidas de protección lo que beneficia evitar el contagio tanto de las mismas enfermeras como de los pacientes neonatos.
\end{abstract}

Palabras clave: Servicio de Neonatología, medidas de bioseguridad, medidas de protección, personal de enfermería, riesgos de contagio, riesgos laborales.

\begin{abstract}
The qualified investigation Biosecurity and protection measures for nurses in the neonatology service of the Regional Maternal and Child Teaching Hospital El Carmen de Huancayo, part the following problem being formulated: In what measure does the level of knowledge on bioseguridad relate to the use of protection measures of the nurses in the Service of Neonatalogy of the Educational Mother Infantile Hospital " Carmen " of Huancayo?; the aim that it chases is: To determine the relation between the level of knowledge on bioseguridad and the use of protection measures of the nurses in the Service of Neonatalogy of the Educational Mother Infantile Hospital "El Carmen" of Huancayo.

The design of investigation is correlacional, explanatory level, basic type, the used method has been historically and descriptively. The sample is shaped by 40 nurses (you). The results of the social, empirical investigation have allowed us to elaborate important conclusions like that there is a high knowledge of knowledge on the measures of bioseguridad on the part of the nurses of the Service of Neonatalogy of the Educational Mother Infantile Hospital " Carmen " of Huancayo, this level of knowledge on the measures of bioseguridad is related significantly to the employment of protection measures what he benefits to avoid the contagion both of the same nurses and of the patients neonatals.
\end{abstract}

Key words: Service of Neonatalogy, measures of bioseguridad, measures of protection, personnel of infirmary, risks of contagion, labor risks. 


\section{INTRODUCCIÓN}

Actualmente en el mundo se está observando, con mucho interés, la salud ocupacional del personal que labora en las instituciones sanitarias, debido a que en los últimos años, el comportamiento del trabajador ha ido modificándose a medida que se han producido avances en la ciencia y tecnología; de tal manera, que la salud del trabajador de ciencias de la salud cobra un papel predominantemente importante para las instituciones.

La salud del trabajador hospitalario que se encuentran en constante relación con enfermedades transmisibles, depende en gran medida de las condiciones de trabajo que brinden estos, como también del conocimiento que tengan acerca de bioseguridad y la puesta en práctica de las medidas de protección en su trabajo diario; también, las arduas horas consecutivas de trabajo, pueden repercutir en la calidad de atención al paciente afectándolo económica y socialmente, con consecuente impacto económico, social y altos costos para la institución, si estas no son llevadas de manera correcta.

Los trabajadores de salud están expuestos a múltiples riesgos ocupacionales, principalmente biológicos, por encontrarse en contacto con pacientes que padecen enfermedades infectocontagiosas y con objetos punzocortantes contaminados con sangre y otros fluidos corporales; es por esto, que ha sido identificada como la causa más frecuente por la cual el personal de salud se contamina con sangre o fluidos corporales infectocontagiosos.

El tema de infecciones intrahospitalarias no es reciente. A pesar de las recomendaciones realizadas por los organismos internacionales y nacionales de salud, los trabajadores de salud siguen accidentándose y realizando sus tareas, no siempre, de la manera más segura. Una de las razones principales para que esto suceda, es que cada hospital tiene sus propios factores de riesgo para poder implantar programas de prevención. La prevención de enfermedades y accidentes ocupacionales está dada por la aplicación de las medidas de bioseguridad: universalidad y precauciones estándar. La bioseguridad es entendida como el conjunto de medidas preventivas para proteger la salud y seguridad de las personas en el ambiente hospitalario frente a riesgos biológicos, físicos, químicos, psicológicos y mecánicos.

La exposición ocupacional de trabajadores de salud en áreas críticas que utilizan agujas y pueden estar expuestos a contaminantes biológicos, incrementa el riesgo de sufrir lesiones por pinchazos y otros. Este tipo de lesiones son frecuentes y pueden provocar infecciones graves o mortales causados por agentes patógenos presentes en la sangre, tales como el virus de la hepatitis B, $\mathrm{C}$ o el virus de la Inmunodeficiencia Humana; sin considerar, otros virus y enfermedades producidas por diferentes microorganismos.
La enfermería constituye una profesión que requiere de una profunda entrega y sacrificio a favor del paciente, con un alto sentido del humanismo y la responsabilidad que harán de la enfermera un ser humano más sensible y más capaz de enfrentarse a todas las adversidades y dificultades que se presenten durante su camino como profesional. En toda institución de salud, se encuentran las medidas de bioseguridad, que se consideran muy importantes dentro de área epidemiológica, y que están orientadas a proteger la salud del personal, paciente y comunidad; cuyo objetivo es disminuir el riesgo de transmisión de enfermedades, tanto para el paciente, como para el personal durante el cumplimiento de sus funciones.

Los hospitales son instituciones que presentan características especiales, entre ellos, la abundante presencia de gérmenes, quienes pueden colonizar o infectar al ser humano. El personal que trabaja en un hospital está en permanente contacto con microorganismos de todo tipo: virus, bacterias, hongos y parásitos; a pesar de ello, no es frecuente que presenten infecciones debido a las capacidades intrínsecas de defensa (como huésped), a las características del medio donde se encuentra y a las características intrínsecas de los microorganismos al que está expuesto.

La labor de enfermería está sustentada en el cuidado de la salud, el cual es un proceso interactivo entre una persona que tiene necesidad de ayuda y otra capaz de ofrecérsela. En este interactúan sentimientos, emociones, creencias, valores, conocimientos, experiencias y vivencias de salud anteriores. En tal sentido, la enfermería es la ciencia del cuidado de la salud del ser humano; es por ello, que la labor del profesional de enfermería es muy amplia, debido a que además de profesionalismo, capacitación y entereza, requieren de una gran paciencia para tratar adecuadamente a los pacientes. Son ellas quienes en ocasiones pasan más tiempo con un enfermo, ya sea en hospitales o en el propio hogar del paciente.

En esta perspectiva, cuando hablamos de conocimiento, nos referimos al conjunto de ideas, conceptos, enunciados que pueden ser claros, precisos, ordenados e inexactos, en base a ello se tipifica al conocimiento en: científico y vulgar. El primero se identifica como conocimiento racional, analítico, objetivo y sistemático a través de la experiencia y; el conocimiento vulgar, como un conocimiento vago, inexacto, limitado a la observación.

Otra de las variables a estudiar es el de bioseguridad, la cual se define como el término empleado para reunir y definir las normas relacionadas con el comportamiento preventivo del personal del hospital, frente a riesgos propios de sus actividades diarias. Pero hace relación también al conjunto de normas, disponibilidades y facilidades que la institución tiene permanentemente actualizadas para evitar cualquier riesgo físico o psicológico del personal que labora dentro de la institución, igual que los usuarios.

Podemos decir, que la seguridad biológica o bio- 
seguridad, es el término utilizado para referirse a los principios y prácticas aplicadas, con el fin de evitar la exposición no intencional a patógenos y toxinas. Hablar de bioseguridad, es enfatizar en las medidas preventivas pertinentes a los riesgos biológicos para proteger la salud y la seguridad del personal que trabaja en cualquier institución hospitalaria. Las normas de bioseguridad están destinadas a reducir el riesgo de transmisión de microorganismo, de fuentes reconocidas o no reconocidas, de infección en servicios de salud, vinculadas a accidentes por exposición a sangre y fluidos corporales.

Se espera que se continúe en el desarrollo de temas, como el tratado, y que sirva como aporte a la ciencia jurídica. Asimismo, se espera contribuir al conocimiento de la realidad y que las propuestas, de la presente investigación, ayuden a mejorar y propicien un mayor estudio a través de equipos multidisciplinarios para que los aportes puedan ser aplicados en beneficio de la familia y de la sociedad en general.

\section{MATERIALES Y MÉTODOS}

\section{Lugar de ejecución}

Hospital Regional Docente Materno Infantil El Carmen de Huancayo - Región Junín.

\section{Población}

Está conformada por 40 enfermeras y enfermeros que laboran en el Hospital D.A, Carrión de Huancayo - Región Junín. Se trabajó con toda la población por ser finita.

\section{Objetivos}

\section{a. Objetivo General}

Determinar la relación entre el nivel de conocimiento sobre bioseguridad y el uso de medidas de protección de las enfermeras en el Servicio de Neonatología del Hospital Docente Materno Infantil "El Carmen" de Huancayo.

\section{b. Objetivos específicos}

Establecer la relación entre el nivel de conocimiento sobre bioseguridad con la seguridad del paciente a cargo de las enfermeras en el Servicio de Neonatología del Hospital Docente Materno Infantil "El Carmen" de Huancayo.

Establecer la relación entre el uso de las medidas de protección de las enfermeras con la reducción de riesgos de contagios en el Servicio de Neonatología del Hospital Docente Materno Infantil “El Carmen” de Huancayo.

\section{Métodos}

\section{Método de Investigación}

La investigación ha utilizado como método general el método científico y de forma específica el histórico y descriptivo.

Es una investigación descriptivo y explicativo: Porque se ha buscado especificar las propiedades de las variables independientes o factores (descriptivo) que determinan el tratamiento que se le da a los riesgos laborales del personal de enfermería de Sala de Operaciones (explicativo).

\section{Diseño de investigación}

Correlacional

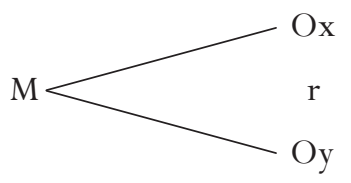

Dónde:

$M \quad$ : Muestra

$O X$ : Observación realizada a $\mathrm{V}$. conocimientos de bioseguridad.

Oy : Observación realizada a la V. medidas de protección de las enfermeras.

$R \quad$ : Relación que existe entre las variables sometidas a estudio.

\section{Tipo de investigación}

La investigación ha de ser básica, por proponerse conocer los antecedentes de la bioseguridad y las medidas de protección de las enfermeras en el servicio de neonatología del Hospital Docente Materno Infantil “El Carmen” de Huancayo; así como, la relación que se establecen entre ambas variables para hacer y para actuar. Ello permitirá construir y realizar una propuesta que busque modificar la situación actual en el tema de riesgos laborales.

\section{Procesamiento de datos}

La investigación responde a un diseño de recolección de datos que se ha aplicado a toda la población, realizado al personal de enfermería.

Asimismo, se ha utilizado Microsoft Excel para el procesamiento de la información, así como para la conversión en tablas.

Dentro del análisis estadístico se ha utilizado la tabla de contingencia aplicando el contraste del Chi2 con un nivel de significancia del 95\% $(p<0.05)$.

\section{Plan de análisis e interpretación de datos}

\section{Análisis descriptivo}

El análisis del trabajo ha sido descriptivo y se ha realizado teniendo en cuenta las hipótesis, basándose en datos estadísticos obtenidos a través de la muestra seleccionada y que ha servido para dar la interpretación adecuada.

\section{Análisis inferencial}

Los datos obtenidos, han permitido deducir las ideas centrales; para lo cual, se han aplicado los principios básicos de la estadística inferencial para contrastar las hipótesis según el tipo de variables. 


\section{Análisis correlacional}

El mismo que se ha desarrollado buscando establecer la relación entre las variables, identificarlas y definir los cambios que afectan a la otra.

\section{Análisis explicativo}

Se ha desarrollado describiendo las variables que integran la hipótesis y se determinó las relaciones que existen entre ellas, persiguiendo establecer qué es lo que da origen a la casualidad que prima en las relaciones de las variables, definir por qué una variable determina a la otra, a través de la fórmula de causa-efecto.

\section{Paquetes estadísticos}

Para el análisis estadístico se ha empleado el SPSS22 y el paquete MS Excel.

\section{RESULTADOS}

Para el desarrollo de la presente investigación se ha aplicado un cuestionario dirigido a todas las enfermeras que trabajan en el Servicio de Neonatología del Hospital Regional Docente Materno Infantil El Carmen de Huancayo, cuyos resultados han servido para demostrar las hipótesis planteadas; así como, para lograr los objetivos. Sobre la primera pregunta acerca del principio básico de la bioseguridad nos contestaron de la siguiente forma y se presenta en la tabla.

Tabla $\mathbf{N}^{\circ}$ 01. Principio básico de la bioseguridad

\begin{tabular}{|c|c|c|}
\hline Alternativa & Cantidad & $\%$ \\
\hline No contagiar al paciente & 04 & 10 \\
\hline No contagiarse & 02 & 05 \\
\hline No me contagio y no contagio & 32 & 80 \\
\hline No me contagio de infecciones & 02 & 05 \\
\hline A y $C$ & 00 & 00 \\
\hline TOTAL & 40 & 100 \\
\hline
\end{tabular}

Fuente: Elaborada en base a cuestionario.

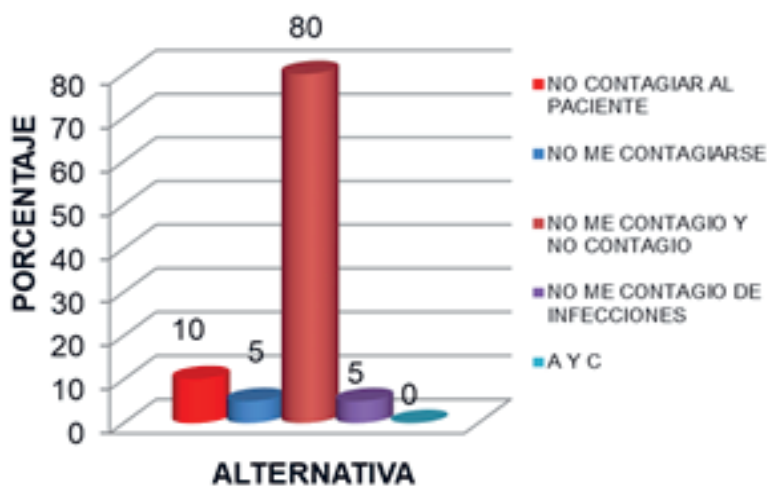

Figura 1. Principio básico de la bioseguridad

Como se puede ver, en la tabla $\mathrm{N}^{\circ} 1$, el $80 \%$ del personal de enfermería que trabaja en el Servicio de Neonatología del
Hospital Regional Docente Materno Infantil El Carmen de Huancayo, señalan como principio básico de seguridad el no contagiarse y no contagiar a los pacientes, siendo lo correcto. Un 10\% señala que es no contagiar al paciente, un 5\% no contagiarse y otro $5 \%$ no me contagio de infecciones.

La siguiente pregunta, es sobre la definición de lo que son la medidas de bioseguridad habiendo contestado de la siguiente forma:

Tabla $\mathbf{N}^{\circ}$ 02. Definición de las medidas de bioseguridad

\begin{tabular}{lcc}
\hline \multicolumn{1}{c}{ Alternativa } & Cantidad & $\%$ \\
\hline $\begin{array}{l}\text { Conjunto de medidas preventivas } \\
\text { que protegen la salud y la seguri- } \\
\text { dad del personal frente a riesgos } \\
\text { laborales producidas por agentes } \\
\text { biológicos, físicos o químicos. }\end{array}$ & 38 & 95 \\
$\begin{array}{l}\text { Conjunto de normas para evitar la } \\
\text { propagación de enfermedades e } \\
\text { interumpir el proceso de transmi- } \\
\text { sión de infecciones. }\end{array}$ & 00 & 00 \\
$\begin{array}{l}\text { Conjunto de medidas para elimi- } \\
\text { nar, inactivar o matar gérmenes } \\
\text { patógenos. }\end{array}$ & 00 & 00 \\
$\begin{array}{l}\text { Conjunto de medidas de protección } \\
\text { del personal y del ambiente de traba- } \\
\text { jo mediante la utilización de las medi- } \\
\text { das de bioseguridad y el uso de equi- } \\
\text { pos de seguridad apropiada frente a } \\
\text { potenciales agentes infecciosos. }\end{array}$ & 02 & 05 \\
\begin{tabular}{l} 
Todas las anteriores. \\
\multicolumn{1}{c}{ Total }
\end{tabular} & \\
\hline
\end{tabular}

Fuente: Elaborada en base a cuestionario.

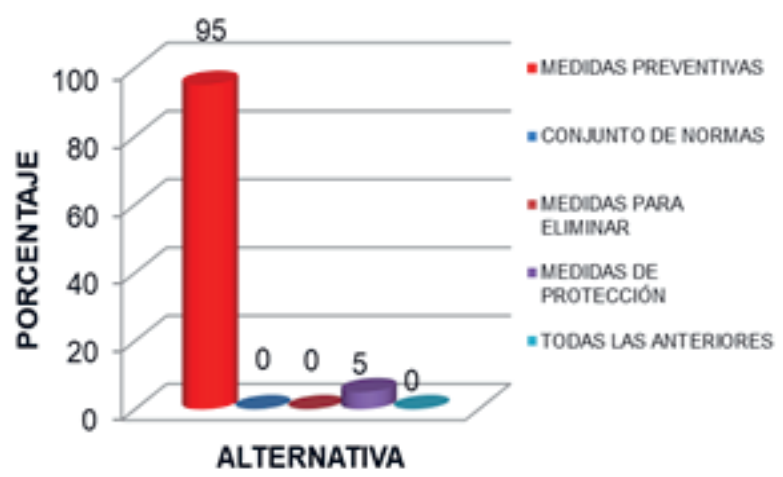

Figura 2. Definición de las medidas de bioseguridad

Se aprecia que el 95\% respondió acertadamente, al definir que las medidas de bioseguridad son el conjunto de medidas preventivas que protegen la salud y la seguridad del personal frente a riesgos laborales producidas por agentes biológicos, físicos o químicos,. Sólo el 5\% manifestó que son el conjunto de medidas de protección del personal y del ambiente de trabajo mediante la utilización de las medidas de bioseguridad y el uso de equipos de seguridad apropiada frente a potenciales agentes infecciosos. La siguiente pregunta se refiere a los principios de la bioseguridad, con cuyas respuestas se ha elaborado la siguiente tabla: 
Tabla $\mathbf{N}^{\circ}$ 03. Prinicipio de la bioseguridad

\begin{tabular}{lcc}
\hline \multicolumn{1}{c}{ Alternativa } & Cantidad & \% \\
\hline $\begin{array}{l}\text { Protección, aislamiento, univer- } \\
\text { salidad. }\end{array}$ & 00 & 00 \\
$\begin{array}{l}\text { La universalidad, uso de barreras } \\
\text { protectoras, control de residuo }\end{array}$ & 32 & 80 \\
$\begin{array}{l}\text { Barreras protectoras, universali- } \\
\text { dad, control de infecciones. }\end{array}$ & 00 & 00 \\
$\begin{array}{l}\text { La universalidad, uso de barreras } \\
\text { de protección y manejo de resi- } \\
\text { duos sólidos. }\end{array}$ & 08 & 20 \\
$\begin{array}{l}\text { A y B } \\
\text { Total }\end{array}$ & $\mathbf{4 0}$ & $\mathbf{1 0 0}$ \\
\hline \hline
\end{tabular}

Fuente: Elaborada en base a cuestionario

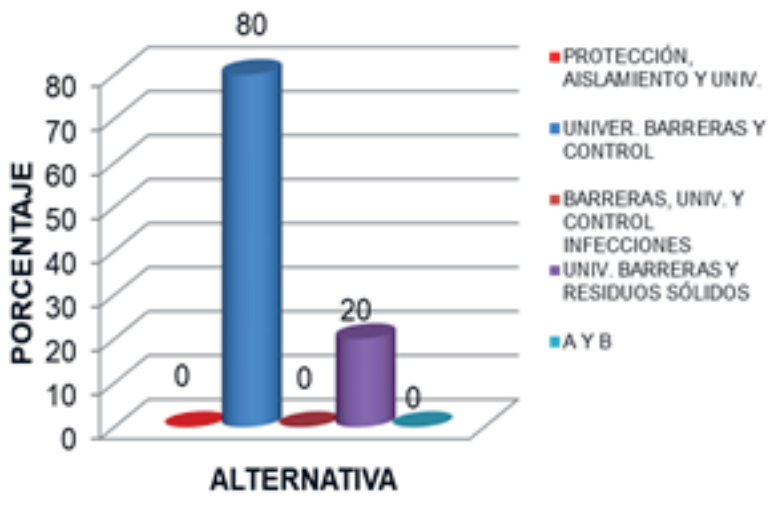

Figura 3. Principios de bioseguridad

Tenemos en el cuadro anterior que el $80 \%$ de los enfermeros consultados manifiesta en forma correcta que los principios de la bioseguridad son la universalidad, el uso de barreras protectoras y el control de residuo; en tanto que, el $20 \%$ opina que son: la universalidad, el uso de barreras protectoras y el manejo de residuos sólidos, habiéndose confundido, al no sólo ser el manejo de residuos sólidos, sino también, líquidos y gaseosos.

A continuación, se les consultó acerca de qué barreras protectoras primarias permiten evitar el contacto físico de fluidos potencialmente peligrosos, con cuyas respuestas se construyó el siguiente gráfico.

Tabla $\mathbf{N}^{\circ}$ 04. Barreras protectoras primarias para evitar el contacto físico de fluidos peligrosos

\begin{tabular}{|c|c|c|}
\hline Alternativa & Cantidad & $\%$ \\
\hline Guantes y mascarillas & 38 & 95 \\
\hline Protección ocular y tapaboca & 02 & 05 \\
\hline Lentes & 00 & 00 \\
\hline Protección de los pies & 00 & 00 \\
\hline Sólo A y C & 00 & 00 \\
\hline Total & 40 & 100 \\
\hline
\end{tabular}

Fuente: Elaborada en base a cuestionario

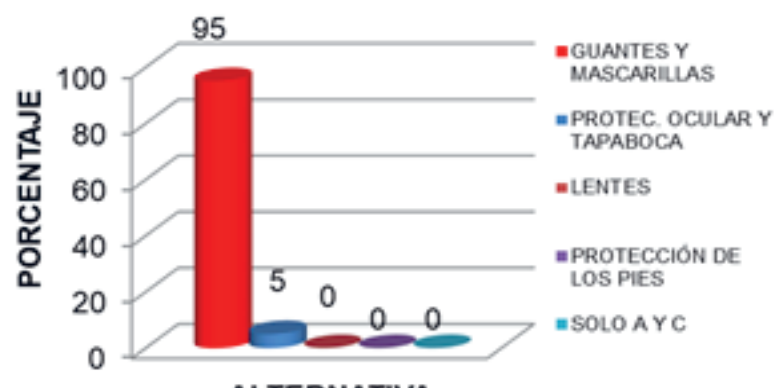

ALTERNATIVA

Figura 4. Barreras protectoras primarias para evitar el contacto de fluidos peligrosos

Se puede observar que el 95\% del personal de enfermería consultado, manifiesta que las barreras protectoras primarias que permiten evitar el contacto físico de fluidos potencialmente peligrosos son los guantes y mascarillas, mientras que sólo un 5\% opina que es la protección ocular y tapaboca.

Se les consultó acerca de cuáles son las precauciones universales de bioseguridad, y contestado de la siguiente manera:

Tabla $\mathbf{N}^{\circ} 05$. Precauciones universales de bioseguridad

\begin{tabular}{lcc}
\hline \multicolumn{1}{c}{ Alternativa } & Cantidad & $\%$ \\
\hline $\begin{array}{l}\text { Lavado de manos después del } \\
\text { contacto con paciente, vacuna- } \\
\text { ción anual, uso de botas, uso de } \\
\text { guantes. }\end{array}$ & 02 & 05 \\
$\begin{array}{l}\text { Lavado de manos, control de va- } \\
\text { cunación, uso de mandilón, evitar } \\
\text { salpicaduras, uso de chaqueta. }\end{array}$ & 02 & 05 \\
$\begin{array}{l}\text { Uso de guantes, lavado de manos } \\
\text { antes del contacto con paciente, }\end{array}$ & 02 & 05 \\
$\begin{array}{l}\text { uso de mandilón, uso de lentes } \\
\text { protectoras, control de vacunación. }\end{array}$ & & \\
$\begin{array}{l}\text { Lavado de manos antes y después } \\
\text { del contacto con paciente, uso de } \\
\text { guantes, uso de mascarilla, uso de } \\
\text { mandilón, uso de botas, vacuna- } \\
\text { ción anual. }\end{array}$ & 32 & 80 \\
B y C & 02 & 05 \\
\hline \multicolumn{1}{c}{ Total } & 40 & 100 \\
\hline \hline
\end{tabular}

Fuente: Elaborada en base a cuestionario

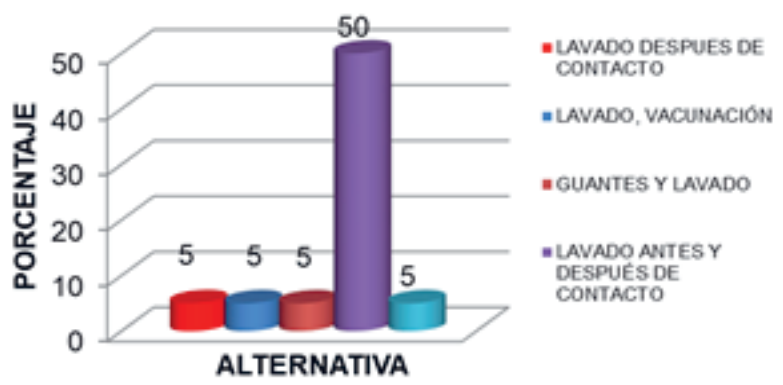

Figura 5. Barreras protectoras primarias para evitar el contacto de fluidos peligrosos 
Como se puede vislumbrar del cuadro precedente, para el 32\% de los enfermeros las precauciones universales de bioseguridad son el lavado de manos antes y después del contacto con paciente, uso de guantes, uso de mascarilla, uso de mandilón, uso de botas, vacunación anual, lo cual es correcto; para un 5\% son el lavado de manos después del contacto con paciente, vacunación anual, uso de botas y uso de guantes; para un 5\%, vienen a ser el lavado de manos, control de vacunación, uso de mandilón, evitar salpicaduras y uso de chaqueta. Para otro 5\% es el uso de guantes, lavado de manos antes del contacto con paciente, uso de mandilón, uso de lentes protectoras, control de vacunación; y finalmente, para un 5\% son las respuestas b y c.

Con lo cual se tiene que un promedio que se ha podido deducir por los resultados de los cuadro anteriores es el siguiente:

Tabla $\mathbf{N}^{\circ}$ 06. Nivel de conocimientos sobre bioseguridad de las enfermeras del servicio de neonatología

\begin{tabular}{|c|c|c|}
\hline ALTERNATIVA & Cantidad & $\%$ \\
\hline Muy alto & 00 & 00 \\
\hline Alto & 38 & 95 \\
\hline Medio & 02 & 05 \\
\hline Bajo & 00 & 00 \\
\hline Muy bajo & 00 & 00 \\
\hline TOTAL & 40 & 100 \\
\hline
\end{tabular}

Fuente: Elaborado en base a cuestionario.

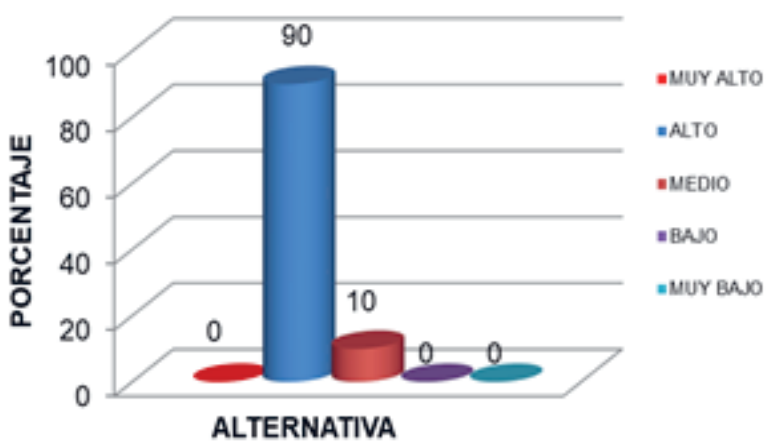

Figura 6. Nivel de conocimiento sobre bioseguridad

Tenemos como promedio general, que el nivel de conocimiento sobre bioseguridad, que las enfermeras del Servicio de Neonatología del Hospital Regional Docente Materno Infantil El Carmen de Huancayo, es en un 90\% alto, lo que nos lleva a comprender que las enfermeras y enfermeros se encuentran los suficientemente capacitados en cuando a la comprensión teórica y académica de lo que viene a ser la bioseguridad, la forma como se debe manejar las medidas de bioseguridad, los principios, los manuales y directivas emanadas del MINSA. Sólo un 10\% tiene un nivel de conocimiento medio sobre la bioseguridad.
La siguiente pregunta que se formuló, se refiere a cuándo es indispensable para la atención del neonato el uso de guantes limpios, cuyas respuestas son de la siguiente manera.

Tabla $\mathbf{N}^{\circ} \mathbf{0 7}$. Cuando es indispensable el uso de guantes limpios

\begin{tabular}{lcc}
\hline \multicolumn{1}{c}{ Alternativa } & Cantidad & $\%$ \\
\hline Hay contacto con fluidos corporales & 02 & 05 \\
$\begin{array}{l}\text { El personal de Salud presenta le- } \\
\text { siones en la piel }\end{array}$ & 02 & 05 \\
Se realiza el baño del recién nacido. & 02 & 05 \\
Se realicen procedimientos invasivos & 04 & 10 \\
A y B & 30 & 75 \\
\hline Total & 40 & 100 \\
\hline
\end{tabular}

Fuente: Elaborado en base a cuestionario.

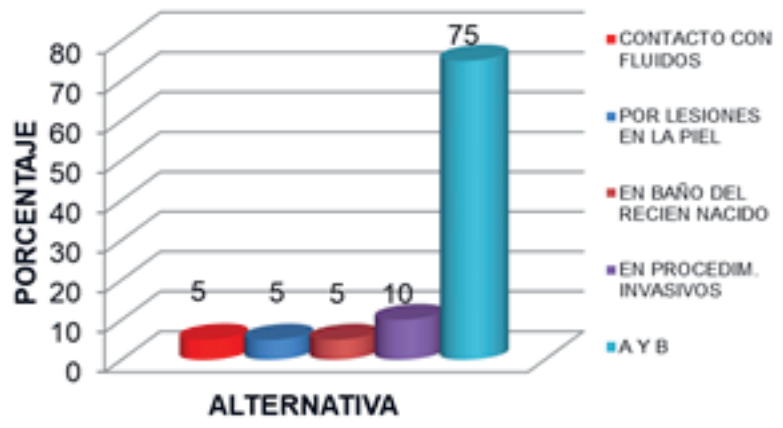

Figura 7. Uso indispensable de guantes limpios

Se aprecia que, para el 75\% de los consultados, el uso de los guantes limpios es indispensable para la atención del neonato cuando hay contacto con fluidos corporales y cuando el personal de salud presenta lesiones en la piel; un $5 \%$, sólo cuando hay contacto con fluidos corporales, otro $5 \%$ opina cuando el personal de salud presenta lesiones en la piel. Un 5\% manifiesta cuando se realiza el baño del recién nacido y un 10\% cuando se realizan procedimientos invasivos.

Otra de las preguntas se refiere a ¿en qué situación cree usted que es necesario el uso de la mascarilla?, cuyas respuestas las vemos en el siguiente cuadro.

Tabla $\mathrm{N}^{\circ} \mathbf{0 8}$. Situación en la que es necesaria el uso de la mascarilla

\begin{tabular}{lcc}
\hline \multicolumn{1}{c}{ Alternativa } & Cantidad & $\%$ \\
\hline $\begin{array}{l}\text { Existe riesgo de salpicadura de flui- } \\
\text { dos y secreciones contaminadas }\end{array}$ & 02 & 05 \\
$\begin{array}{lcc}\text { Durante todo el turno } \\
\begin{array}{l}\text { En la atención de pacientes con in- } \\
\text { fecciones respiratorias. }\end{array}\end{array}$ & 02 & 05 \\
$\begin{array}{l}\text { Sólo para proteger al paciente. } \\
\text { A y C }\end{array}$ & 00 & 00 \\
\hline \hline
\end{tabular}

Fuente: Elaborado en base a cuestionario. 


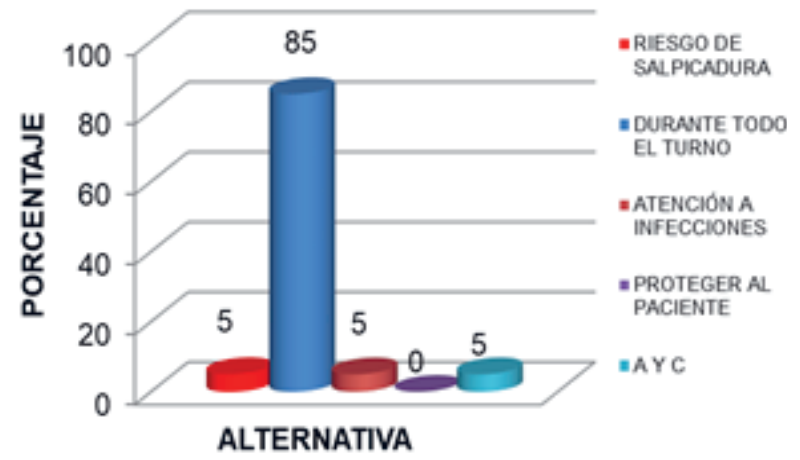

Figura 8. Situación en la que es necesaria el uso de la mascarilla

Se aprecia que un $34 \%$ de los consultados, tiene en cuenta que el uso de la mascarilla es necesaria durante todo el turno, un 5\% opina que cuando existe riesgo de salpicadura de fluidos y secreciones contaminadas, un $5 \%$ manifiesta en la atención de pacientes con infecciones respiratorias y, otro $5 \%$ en dos casos juntos, cuando hay riesgo de salpicadura de fluidos y secreciones contaminadas y en la atención de pacientes con infecciones respiratorias.

Otra de las preguntas que se realizó es sobre cuándo considera que se debe realizar el lavado de las manos, contestándose de la siguiente manera:

Tabla $\mathbf{N}^{\circ}$ 09. ¿Cuándo se debe realizar el lavado de las manos?

\begin{tabular}{lcc}
\hline \multicolumn{1}{c}{ ALTERNATIVA } & Cantidad & $\%$ \\
\hline \hline Antes del contacto con el paciente & 00 & 00 \\
Antes de realizar una tarea aséptica & 00 & 00 \\
$\begin{array}{l}\text { Después del riesgo de exposición a } \\
\text { líquidos corporales }\end{array}$ & 02 & 05 \\
$\begin{array}{l}\text { Después del contacto con el paciente } \\
\text { Todas las anteriores }\end{array}$ & 02 & 05 \\
\hline \multicolumn{1}{|c|}{ TOTAL } & $\mathbf{4 0}$ & $\mathbf{1 0 0}$ \\
\hline
\end{tabular}

Fuente: Elaborado en base a cuestionario.

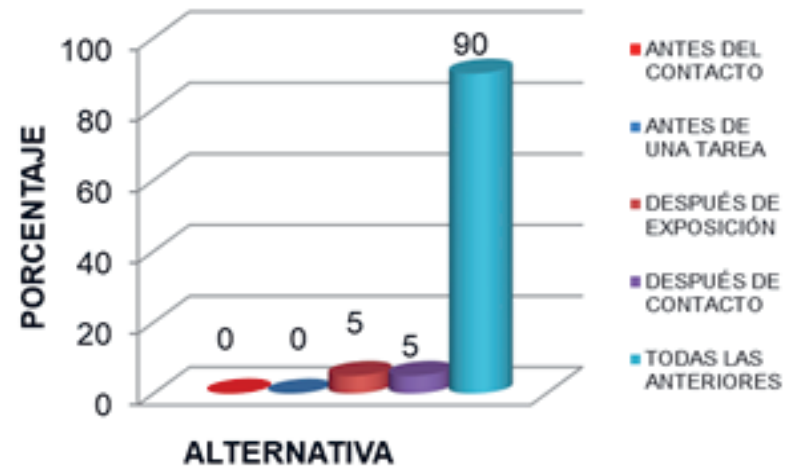

Figura 9. ¿Cuándo se debe realizar el lavado de las manos?

Se tiene que el $90 \%$ realiza el lavado de las manos en todo momento, desde antes del contacto con el paciente hasta después del contacto con el paciente, un 5\% manifiesta después del riesgo a exposición a líquidos corporales y otro 5\% después del contacto con el paciente.

Asimismo, se preguntó si otro elemento apropiado para el lavado de manos se combina con el jabón líquido; habiendo construido el siguiente cuadro con sus respuestas.

Tabla $N^{\circ} 10$ Para el lavado de las manos ¿Se usa el jabón líquido con otro elemento?

\begin{tabular}{|c|c|c|}
\hline Alternativa & Cantidad & $\%$ \\
\hline Yodopovidona & 00 & 00 \\
\hline Gluconato de Clorhexidina al $2 \%$ & 00 & 00 \\
\hline Gluconato de Clorhexidina al $4 \%$ & 00 & 00 \\
\hline Alcohol Puro & 00 & 00 \\
\hline Ninguno & 40 & 100 \\
\hline Total & 40 & 100 \\
\hline
\end{tabular}

Fuente: Elaborado en base a cuestionario.

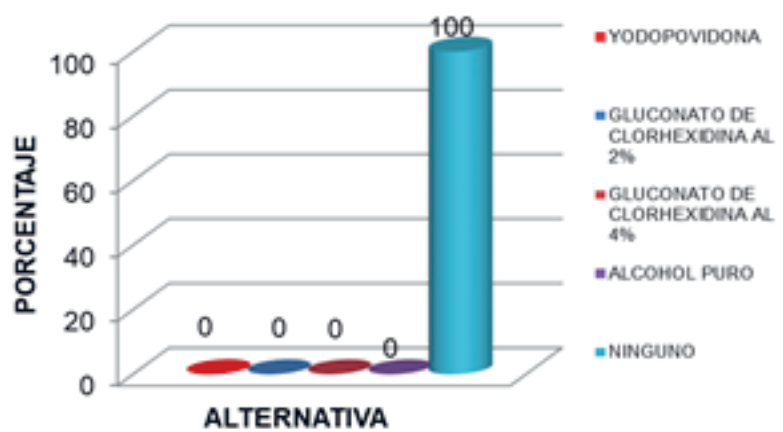

Figura 10. Para el lavado de las manos se usa el jabón líquido con otro elemento

En este caso, se puede ver que la totalidad de los enfermeros y enfermeras, manifiestan que para el lavado de manos se debe usar el jabón líquido, solo sin necesidad de mezclar con otro elemento, ya que por sí sólo es eficiente para dejar las manos limpias de impurezas.

Se les preguntó acerca de cuáles son las barreras protectoras de bioseguridad que viene usando durante el desarrollo de su trabajo en el Servicio de Neonatología, contestando de la siguiente manera.

Tabla $\mathbf{N}^{\circ}$ 11. Barreras protectoras de bioseguridad

\begin{tabular}{|c|c|c|}
\hline Alternativa & Cantidad & $\%$ \\
\hline Mandilón, botas, gorros y guantes & 00 & 00 \\
\hline Mascarilla, bata estéril, gorro y botas & 00 & 00 \\
\hline $\begin{array}{l}\text { Guantes, mandil, mascarillas, gorro } \\
\text { y botas }\end{array}$ & 00 & 00 \\
\hline Mascarilla, mandilón, gorro y guantes & 40 & 100 \\
\hline$B$ y $D$ & 00 & 00 \\
\hline Total & 40 & 100 \\
\hline
\end{tabular}

Fuente: Elaborado en base a cuestionario 


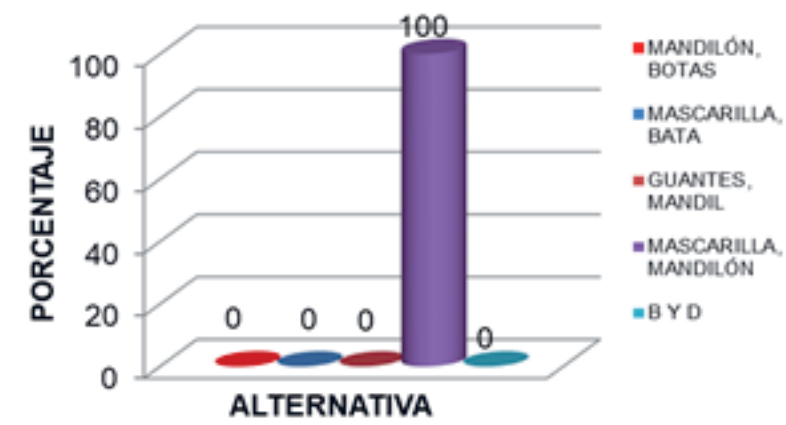

Figura 11. Barreras protectoras de bioseguridad

En este caso, todos contestaron que las barreras protectoras de bioseguridad con la mascarilla, el mandilón, el gorro y los guantes, al ser lo que a diario están usando en el servicio.

También se les preguntó acerca de ¿En qué situaciones está indicado el uso de mandilones?, y contestaron de la siguiente manera.

Tabla $\mathrm{N}^{\circ}$ 12. Situaciones en que está indicado el uso de mandilones

\begin{tabular}{lcc}
\hline \multicolumn{1}{c}{ Alternativa } & Cantidad & $\%$ \\
\hline $\begin{array}{l}\text { En toda sala de hospitalización de } \\
\text { Neonatología y en todo procedi- } \\
\text { miento que implique exposición a } \\
\text { material contaminado }\end{array}$ & 00 & 00 \\
$\begin{array}{l}\text { En todo procedimiento que impli- } \\
\text { que exposición al material no con- } \\
\text { taminado y estéril }\end{array}$ & 00 & 00 \\
$\begin{array}{l}\text { Para la atención directa del paciente } \\
\text { Para la higiene y comodidad del }\end{array}$ & 40 & 100 \\
$\begin{array}{l}\text { paciente, curación de heridas, ac- } \\
\text { tividades de laboratorio }\end{array}$ & 00 & 00 \\
A, B y C & 00 & 00 \\
\hline Total & 40 & 100 \\
\hline
\end{tabular}

Fuente: Elaborado en base a cuestionario

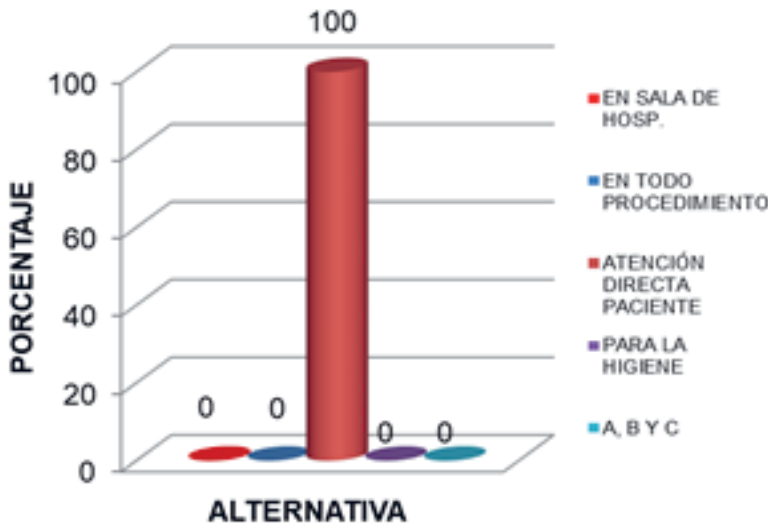

Figura 12. Situaciones en que está indicado el uso de mandilones

Del mismo modo que en las anteriores interrogantes, todos los enfermeros y enfermeras utilizan los mandilones para la atención directa del paciente, al permitirles el poder tener una barrera de protección, tanto para ellos como para los pacientes, y con lo cual están cumpliendo con las normas impartidas por el Ministerio de Salud.
A continuación se tiene la distribución de la aplicación de medidas de protección de bioseguridad por las enfermeras y enfermeros en el Servicio de Neonatología, en base a la práctica cotidiana que tienen las enfermeras encuestadas y que ha servido para poder ver la relación que existe entre el nivel de conocimientos de las medidas de bioseguridad y la aplicación de las medidas de protección sobre bioseguridad.

Tabla $\mathbf{N}^{\circ}$ 13. Cumplimiento de las medidas de protección sobre bioseguridad

\begin{tabular}{lccc}
\hline & Alternativa & Cantidad & $\%$ \\
\hline Cumple & & 40 & 100 \\
No cumple & & 00 & 00 \\
\hline \multirow{2}{*}{ Total } & $\mathbf{4 0}$ & $\mathbf{1 0 0}$ \\
\hline
\end{tabular}

Fuente: Elaborado en base a cuestionario.

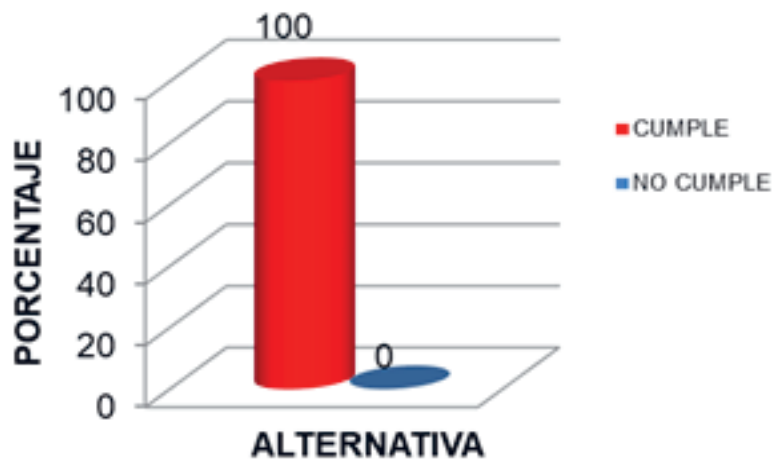

Figura 13. Cumplimiento de las medidas de protección sobre bioseguridad

Se puede deducir que la totalidad del personal de enfermería, que trabajan en el Servicio de Neonatología del Hospital Regional Docente Materno Infantil El Carmen de Huancayo, si cumplen con la práctica diaria de las medidas de protección sobre bioseguridad.

\section{DISCUSIÓN}

En base a los resultados reportados en el desarrollo del trabajo, se deduce que el personal de enfermería que labora en el Servicio de Neonatología del Hospital Regional Docente Materno Infantil El Carmen de Huancayo, tienen en su mayoría, conocimiento acerca del principio básico de seguridad, que es el que no se debe contagiar y no contagiar a los pacientes. Asimismo, la gran mayoría, el 95\% tiene un perfecto conocimiento de los que son la medidas de bioseguridad lo que va a redundar en el buen desarrollo de sus labores dentro del servicio, ya que hablar de bioseguridad es hacer referencia a la vida, a la seguridad necesaria para proteger la existencia de los seres humanos y el cuidado de su salud, para esto existen unas medidas y/o barreras preventivas, y están son las normas básicas de seguridad que nos ayudan a conservar la salud y la vida. 
Por otro lado, el $80 \%$ de los enfermeros conocen bien los principios de bioseguridad, así como, las precauciones universales de bioseguridad. En tanto que, en relación al nivel de conocimiento que tienen los enfermeros del Servicio de Neonatología sobre bioseguridad, es importante destacar que el mayor porcentaje, 95\% tienen un conocimiento alto, mientras que un $5 \%$ tienen un nivel de conocimiento medio acerca de bioseguridad.

La bioseguridad es una doctrina de comportamiento encaminado a lograr actitudes y conductas que disminuyan el riesgo de adquirir enfermedades infectocontagiosas y transmisibles en los trabajadores de salud. El control de enfermedades infectocontagiosas y transmisibles es eficaz, solo si todo el personal de salud que trabaja en ese establecimiento conoce sobre las medidas de bioseguridad y la importancia de su práctica para la atención de los pacientes.

Es importante resaltar que la mayoría de enfermeras adoptan el modelo de Orem, puesto que se fundamenta en la premisa de que ellas tienen la potencialidad para desarrollar sus habilidades intelectuales y prácticas; así como, la motivación esencial para el autocuidado con respecto a las medidas de bioseguridad. El autocuidado es uno de los conceptos centrales de su teoría, como una actividad aprendida por los individuos, orientada hacia un objetivo. Por lo tanto, es una conducta de bioseguridad que se mantiene en situaciones concretas de la vida, dirigida por cada profesional de enfermería hacia sí misma o hacia el entorno.

Por lo cual podemos decir que, es necesario reforzar los conocimientos a través de capacitaciones continuas sobre medidas de bioseguridad, porque los resultados encontrados acerca del nivel de conocimiento, posiblemente sea debido a que el personal de enfermería no recibe constante capacitación; asímismo, puede estar influyendo el tiempo de servicio que tiene cada enfermera En este contexto, las prácticas adecuadas sobre medidas de protección que aplican las enfermeras en el Servicio de Neonatología, reflejan un estado de disposición adquirida y organizada de la propia experiencia que motiva a la enfermera a actuar de una manera característica frente a riesgos intrahospitalarios, puesto que es clave llevar a la práctica, técnicas correctas en los diferentes procedimientos y al fomento de una cultura de prevención orientada hacia el autocuidado, protegiendo de esta manera a los miembros del equipo de salud, al paciente y su familia y, a la comunidad en general.

Si comparamos estos resultados con lo reportado por esta investigación, todavía existe un pequeño número de enfermeras que no cumplen con las medidas de protección. Se observó errores comunes en el departamento, entre ellos se destaca que no se hace uso de mascarillas y guantes limpios o estériles, los cuales no los utilizan a veces por comodidad; pero por otro lado, también por la escases de estos insumos en el servicio en un determinado momento; así mismo, no se usa ropa protectora para manipular fluidos orgánicos o realizar procedimientos invasivos específicos.

Se puede decir, que la Institución tiene a veces, responsabilidad importante en esta parte, al no proveer de manera adecuada los materiales de protección a pesar de las normas existentes. Pero también podría ser que las enfermeras, por la rutina del día a día, olvidan tomar en cuenta sus medidas de protección o simplemente no desean hacerlo, porque no tienen el conocimiento del riesgos que podría traer para ellas y para los neonatos el no cumplir con estas medidas de protección.

En este sentido, se puede determinar que la exposición al riesgo de contaminación por la práctica inadecuada de medidas de protección en el Servicio de Neonatología es preocupante. La falta de continuidad de programas de capacitación y campañas de sensibilización, sobre medidas de bioseguridad y la aplicación de medidas de protección, en este caso, aunada a prácticas inapropiadas de higiene, como deficiente lavado de manos antes y después de los procedimientos, el re-encapuchar las agujas sin precaución, hacen que este grupo profesional este altamente expuesto a enfermedades infectocontagiosas. De todas maneras, no se puede olvidar que el riesgo de infección se reduce si se observan las precauciones universales, estipuladas en los manuales de bioseguridad.

\section{CONCLUSIONES}

- Hay un conocimiento alto de información sobre las medidas de bioseguridad por parte de las enfermeras del Servicio de Neonatología del Hospital Docente Materno Infantil "El Carmen" de Huancayo.

- Este nivel de conocimiento sobre las medidas de bioseguridad se encuentra relacionada significativamente con el empleo de medidas de protección, lo que beneficia evitar el contagio tanto de las mismas enfermeras, como de los pacientes neonatos.

- El nivel de riesgo de contagio del paciente en el Servicio de Neonatología es reducida por cuanto el nivel de conocimiento sobre medidas de bioseguridad, se relaciona significativamente con la seguridad del paciente a cargo de las enfermeras en el Servicio de Neonatología del Hospital Docente Materno Infantil "El Carmen" de Huancayo.

- El uso de las medidas de protección, con que cuentan y practican las enfermeras, ha reducido en forma significativa los riesgos de contagio, tanto para el personal que labora en el Servicio, como para los mismos pacientes.

\section{RECOMENDACIONES}

- Incrementar el ciclo de capacitaciones acerca de las medidas de bioseguridad y medidas de protección, 
haciendo cursos prácticos y con réplicas en los mismos servicios.

- Incrementar el presupuesto del hospital relacionado al abastecimiento de medios y materiales de bioseguridad, con la finalidad de que se encuentren abastecidos y no sufran la falta de ellos.

- Propiciar el intercambio de experiencias entre el personal de los hospitales nacionales y de EsSalud de Junín, a fin de mejorar el nivel de conocimiento y de práctica de las medidas de bioseguridad.

\section{REFERENCIAS BIBLIOGRÁFICAS}

Alarcón, M. y Rubiños, S. Conocimientos y prácticas en la prevención de riesgos biológicos de las enfermeras del Hospital Belén - Lambayeque. 2012. Tesis. Chiclayo. Universidad Católica Santo Toribio de Mogrovejo. 2013.

Becerra, N. Aplicación de las normas de bioseguridad de los profesionales de enfermería. Tesis. Venezuela. Universidad de Oriente. Núcleo Bolívar. 2010.

Bunge, M. La ciencia, su método y su filosofía, Editorial Panamericana. Argentina. 2008.

Burns N, y Grove S. Investigación en enfermería. 3ra. ed. España: Elsevier; 2001.

Concepción, E. y Viña, S. Bases para el diseño del sistema de capacitación de seguridad e higiene ocupacional y bioseguridad para centros de la industria biotecnológica y farmacéutica. Facultad de Ingeniería Industrial La Habana, Cuba $\mathrm{N}^{\circ}$ 18. Cuba. 1998.

Cuyubamba, N. Conocimientos y actitudes del personal de salud, hacia la aplicación de las medidas de bioseguridad del Hospital “Félix Mayorca Soto”Tarma - 2003. Tesis. Lima. UNMSM. 2004.

Durán, M. Teoría de enfermería ¿un camino de herradura?. Aquichan Vol.7. N $^{\circ}$ 2. Colombia. 2007.

Fatone, V. Lógica y teoría del conocimiento, Ed. Kapelusz. México. 2008

Fawcett, J. Conocimiento contemporáneo de enfermería: Análisis y evaluación de modelos y teorías de enfermería. 2da. Edición. Boston. Universidad de Massachusetts. 2005.

Díaz, E. y Heler, M. El conocimiento científico, Vol. 1 y 2. Ed. Universitaria, Buenos Aires. 2005

Godall M. La formación superior en enfermería del profesorado enfermero de las escuelas universitarias de enfermería. Enfermería Clínica. España. 2003.

Hernández A, y Guardado C. La enfermería como disciplina profesional holística. Revista Cubana de Enfermería. Cuba. 2004

Jurado, W.; Solís, S. y Soria, C. Medidas de bioseguridad que aplica el profesional de Enfermería y su relación con la exposición al riesgo laboral en el Hospital Santa María del Socorro, año 2013 - 2014. Tesis. Universidad Nacional
San Luis Gonzaga. Ica. 2014.

Instituto Nacional de Higiene Epidemiología y Microbiología Rev. Cubana. Vol1. Cuba. 2003.

MINSA. Manual de organización y funciones del Departamento de Enfermería. Ministerio de Salud. Lima. 2014.

MINSA. Plan Nacional de Seguridad del Paciente 2013-2015. Ministerio de Salud. Lima. 2013.

MINSA. Norma técnica de prevención y control de infecciones intrahospitalarias. Dirección General de Salud de las Personas, Dirección Ejecutiva de Servicios de Salud. Lima. 2004.

Oficina de Epidemiología y Salud Ambiental-HNDM, Guía básica de bioseguridad hospitalaria, Hospital Nacional Dos de Mayo Lima, Perú 2004.

Oficina General de Epidemiología. Protocolo para el estudio de conocimientos, actitudes y prácticas del personal de salud en el control de las infecciones intrahospitalarias. OGE-RENACE/VIGIA. Lima: Grafica Bellido, 2000.

OPS. Manual de salud ocupacional. Washington DC. OPS. 2002.

Organización Mundial de la Salud (OPS-OMS). Manejo de residuos sólidos hospitalarios en países en desarrollo. Informe de consultoría. Ginebra: OMS; 1992.

Panimboza, C. y Pardo, L. Medidas de bioseguridad que aplica el personal de enfermería durante la estancia hospitalaria del paciente. "Hospital Dr. José Garcés Rodríguez". Salinas 2012-2013. Tesis. Ecuador, Universidad Estatal Península de Santa Elena. 2013.

Salazar, Y. Conocimientos del personal de enfermería sobre las medidas de bioseguridad en las técnicas de administración de medicamentos. Tesis. Guatemala. Universidad San Carlos de Guatemala. 2008.

Wolff, LL. Fundamentos de enfermería. México, 2da. Edición. Edit. Tierra Firme S.A. de C.V. 2002. 